\title{
Easy Vortex Motion in an Artificial Channel of $\mathrm{YBa}_{2} \mathrm{Cu}_{3} \mathrm{O}_{7-\delta}$ Superconducting Films
}

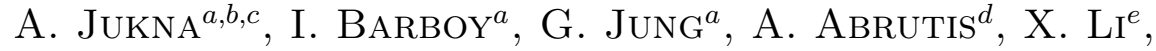 \\ D. WANG ${ }^{e}$ AND R. SobOLEWSKI ${ }^{e}$ \\ ${ }^{a}$ Department of Physics, Ben-Gurion University of the Negev \\ 84105 Beer-Sheva, Israel \\ ${ }^{b}$ High-Tc-Superconductivity Lab., Semiconductor Physics Institute \\ 01108 Vilnius, Lithuania \\ ${ }^{c}$ Department of Physics, Faculty of Natural Sciences \\ Vilnius Gediminas Technical University \\ LT-10223, Vilnius, Lithuania \\ ${ }^{d}$ Department of General and Inorganic Chemistry \\ Vilnius University, LT-03225 Vilnius, Lithuania \\ ${ }^{e}$ Department of Electrical Engineering and Laboratory for Laser Energetics \\ University of Rochester, Rochester, NY 14627, USA \\ Quasi-Josephson effect produced by a coherent vortex motion in the \\ horizontal part of the laser-performed $\Pi$-shaped channel of a $\mathrm{YBa}_{2} \mathrm{Cu}_{3} \mathrm{O}_{7-\delta}$ \\ superconducting bridge was investigated by means of electric transport mea- \\ surements. We observed that in our structures, in a limited range of tem- \\ peratures and bias currents, the vortices were confined in the channel only \\ and moved coherently with the velocity of $3 \times 10^{4} \mathrm{~m} / \mathrm{s}$. The corresponding \\ current-voltage characteristics of the bridge exhibited Josephson-like voltage \\ steps with the amplitude dependent on temperature, but independent of the \\ bias current.
}

PACS numbers: 74.25.Sv, 74.25.Qt, 74.78.Bz

\section{Introduction}

A self-magnetic field of the bias current can penetrate a superconducting bridge in a form of magnetic vortices and antivortices, each embracing the flux quantum $\Phi_{0}=2.07 \times 10^{-15} \mathrm{~Wb}$, at temperatures below the critical temperature $T_{\mathrm{c}}$ of the superconductor. Vortices and antivortices nucleate at opposite edges of a bridge, move towards its center, and annihilate. Motion of current-induced vortices 
is responsible for dissipation and appearance of a resistive state in superconductors at the zero applied magnetic field.

Current-driven vortices move coherently when they are tightly squeezed and strongly interact between themselves [1], or when they interact with a spatiallyperiodic pinning landscape [2]. Josephson-like effects are the hallmark of the coherent vortex motion. A spectacular manifestation of these effects is the appearance of current steps on the current-voltage $(I-V)$ characteristics of microwave (MW) irradiated bridges [3]. The steps appear at voltages at which the inverse of the vortex time-of-flight across the bridge coincides with one of the harmonics of the incident MW radiation. However, the self-Josephson-like steps appear even in the absence of the MW illumination, when the inverse of the time-of-flight of vortices across the half-width of the bridge matches the frequency of the vortex nucleation at the edges [2].

We report our studies on motion of vortices in artificial channels of easy vortex motion fabricated in $\mathrm{YBa}_{2} \mathrm{Cu}_{3} \mathrm{O}_{7-\delta}$ (YBCO) bridges, by means of a laserwriting (LW) technique. The coherent vortex motion was confirmed by a observation of Josephson-like current steps on the bridge $I-V$ characteristics.

\section{Experimental setup and measurements results}

A $0.3 \mu$ m-thick, epitaxial YBCO film, grown by a metal-organic chemical vapor deposition (MOCVD) technique on a $\mathrm{LaAlO}_{3}$ substrate, was patterned by a focused optical beam, generated by an Ar-ion laser operating at a power of 2.3$2.8 \mathrm{~W}$, at which the full deoxygenation of the illuminated areas of the YBCO film occurred. For our laser patterning (LP) procedures, the film has been attached to a computer-controlled $X-Y$ translations tage placed in a nitrogen gas atmosphere. The film was moved with a velocity of $5 \mu \mathrm{m} / \mathrm{s}$, perpendicularly to the beam direction. The width of the LP line was limited by the size of our laser beam spot. Typically, our superconducting films were patterned into a set of six, $50 \mu \mathrm{m}$ wide and $100 \mu \mathrm{m}$ long, bridges with large contact areas (see inset in Fig. 1).

Lower dose of the laser power, e.g., $0.3-0.6 \mathrm{~W}$ and scanning velocity of $50 \mu \mathrm{m} / \mathrm{s}$, was used for LW of a $\Pi$-shaped, $5 \mu \mathrm{m}$ wide channel for easy vortex motion. This LW procedure causes partial depletion of oxygen from the film and reduces the strength of the dominant extended pinning sites, creating favorable conditions for the coherent vortex motion in the channel at currents lower than the critical current, $I_{\mathrm{c}}$, of the reference bridge [4].

Our samples were thermally anchored to a $\mathrm{Cu}$ holder inside an He exchangegas cryostat. Resistivity vs. temperature and $I-V$ curves were measured in a 4-probe arrangement at both polarities of the dc bias current to eliminate thermal offsets. All measurements were performed at the zero external magnetic field.

Figure 1 presents the resistivity vs. temperature dependence of a bridge with the LW channel (called the "LW- bridge") and the same dependence of a channel-free reference device (dashed line). The normal-state resistivity of the LW bridge is higher than that of the reference device. Moreover, for the LW-bridge, 


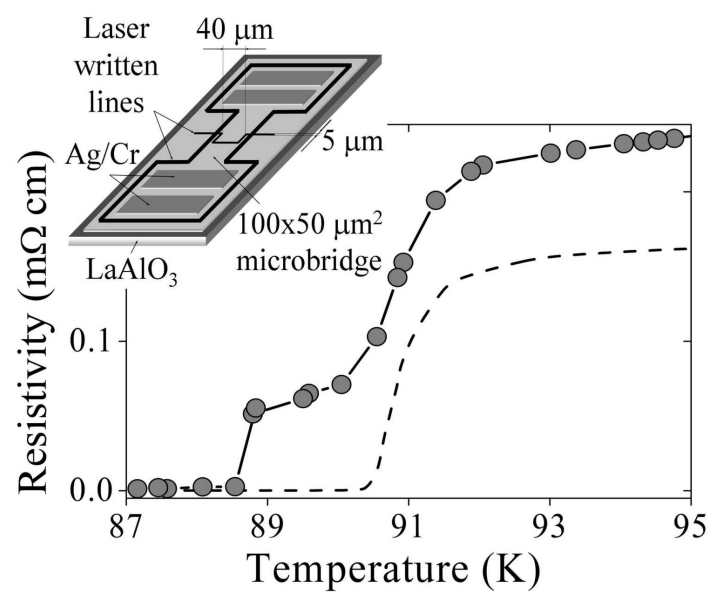

Fig. 1. Resistivity vs. temperature dependence of a YBCO bridge containing a $5 \mu \mathrm{m}$ wide LW-channel (symbols) and resistivity of a reference bridge without the channel (dashed line) at $1 \mu \mathrm{A} \mathrm{dc}$ bias. The inset shows the bridge geometry.

a pronounced, current-dependent, low-temperature resistive tail appers in. The superconducting state, with the transition onset temperature $T_{\mathrm{c}}^{\mathrm{on}}=91.2 \mathrm{~K}$, was not influenced by our LW procedures, as it reflects the properties of the banks of the bridge untreated by LW. Conversely, the device zero-resistivity temperature $T_{\mathrm{c} 0}$ was far below that of the reference device and decreased with the increase in the dc bias current [4].

The $I-V$ curves of the LW-bridge are shown in Fig. 2a and exhibit clear steps in the $T_{\mathrm{c}}^{\mathrm{on}}<T<T_{\mathrm{c} 0}$ temperature range. The threshold current, above which the steps appear, is slightly higher than $I_{\mathrm{c}}$ and increases with the temperature decrease in a different way than $I_{\mathrm{c}}$ of the reference bridge, as seen in Fig. 2b.

Appearance of current steps on the LW-bridge $I-V$ curve can be attributed to the coherent vortex motion and quasi- Josephson effects [2]. If this is the case, the subsequent steps observed on the $I-V$ characteristics should be associated with the entrance of additional vortices into the LW channel. We can estimate the number of vortex-antivortex pairs in the channel from the ratio of dissipation (dynamic resistance) in the vicinity of the $n$-th and $(n+m)$-th step. For a $5 \mu \mathrm{m}$ long and $40 \mu \mathrm{m}$ wide channel at $T=87.8 \mathrm{~K}$ biased with $I=68 \mu \mathrm{A}$ (see inset in Fig. 2a), one can derive the number of vortex pairs $N=36$, as well as the resulting vortex lattice constant $a \approx 1.8 \mu \mathrm{m}$, the effective self-field of the current $\mu_{0} H \approx 0.75 \mathrm{mT}$, and velocity of moving vortices in the horizontal part of the $\Pi$-shaped channel at $3 \times 10^{4} \mathrm{~m} / \mathrm{s}$.

The "voltage height" of a given quasi-Josephson step at a fixed temperature does not increase with the bias current increase [2]. For example, at $T=87.5 \mathrm{~K}$ the averaged step height $\left\langle U_{\text {st }}\right\rangle \approx 7.8 \mu \mathrm{V}$ appears to be constant up to $n=123$ (setup resolution), while the bias current increases from $0.2 \mathrm{~mA}$ up to $0.6 \mathrm{~mA}$. 

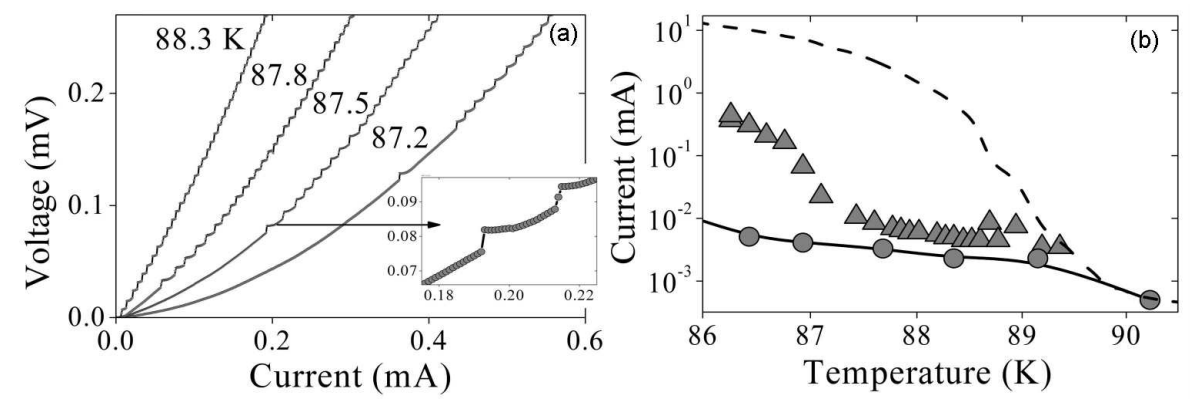

Fig. 2. (a) $I-V$ curves of a YBCO bridge with a $\Pi$-shaped $L W$-channel at $T=87.2$, 87.5, 87.8, 88.3 $\mathrm{K}$ in zero magnetic field. Inset: the first quasi-Josephson step at $87.5 \mathrm{~K}$. (b) The onset current of the coherent vortex motion along this channel vs. temperature (triangles) dependence. For comparison, $I_{\mathrm{c}}$ vs. $T$ dependences of the channeled-bridge (circles) and the reference device (dashed line) are given. The $I_{\mathrm{c}}$ was measured at $10 \mu \mathrm{V}$ criterion.

The latter indicates that the bias current, responsible for the Lorentz force, does not change in the horizontal part of the $\Pi$-shaped channel and is mainly located in the side walks around it. The LW channel exhibits a lower $I_{\mathrm{c}}$ and a weaker critical magnetic field, leading to easy penetration of the current-self magnetic field into this area [4]. However, vortices are driven by an almost constant current density passing the horizontal part of the П-shaped channel.

We have observed a quasi-Josephson effect produced by coherently moving vortices in the horizontal part of the $\Pi$-shaped artificial channel introduced into a YBCO bridge. The channel was produced by LW, which led to partial oxygen removal from laser-activated area of the YBCO stripe. The stepped-like $I-V$ dependences, observed in our bridges, appear in a limited range of temperatures and biasing currents, while the $I_{\mathrm{c}}$ in the channel area is orders of magnitude smaller than the value measured in the laser-untreated YBCO.

\section{Acknowledgments}

This research was supported by the Israeli Science Foundation administered by the Israel Academy of Sciences and Humanities. The work in Rochester was supported by the U.S. AFOSR grant FA9550-06-1-0348, with the additional support provided by the NYSTAR grant to the University of Rochester CAT-EIS Center.

\section{References}

[1] A.A. Lykov, Sov. Phys. Usp. 35, 811 (1992).

[2] Y. Yuzhelevski, G. Jung, C. Camerlingo, M. Russo, M. Ghinovker, B. Ya. Shapiro, Phys. Rev. B 60, 9726 (1999).

[3] L.G. Aslamasov, A. Larkin, Sov. Phys.-JETP 41, 381 (1975).

[4] A. Jukna, I. Barboy, G. Jung, S.S. Banerjee, Y. Myasoedov, V. Plausinaitiene, A. Abrutis, X. Li, D. Wang, R. Sobolewski, Appl. Phys. Lett. 87, 192504 (2005). 